\title{
Manufacturers failed to report infections from duodenoscopes, US investigation finds
}

This News story (BMJ 2016;352:i240, doi:10.1136/bmj.i240) states that investigators estimated outbreaks of infections from duodenoscopes, which occurred in 2013 and 2014, were

responsible for at least 250 deaths. This was incorrect: the investigators estimated that the outbreaks were responsible for at least 250 illnesses - not deaths - due to infections from contaminated duodenoscopes.

Cite this as: BMJ 2016;352:i299

๑ $\odot$ BMJ Publishing Group Ltd 2016 